\title{
La formazione iniziale degli insegnanti di scuola: verso un modello sostenibile
}

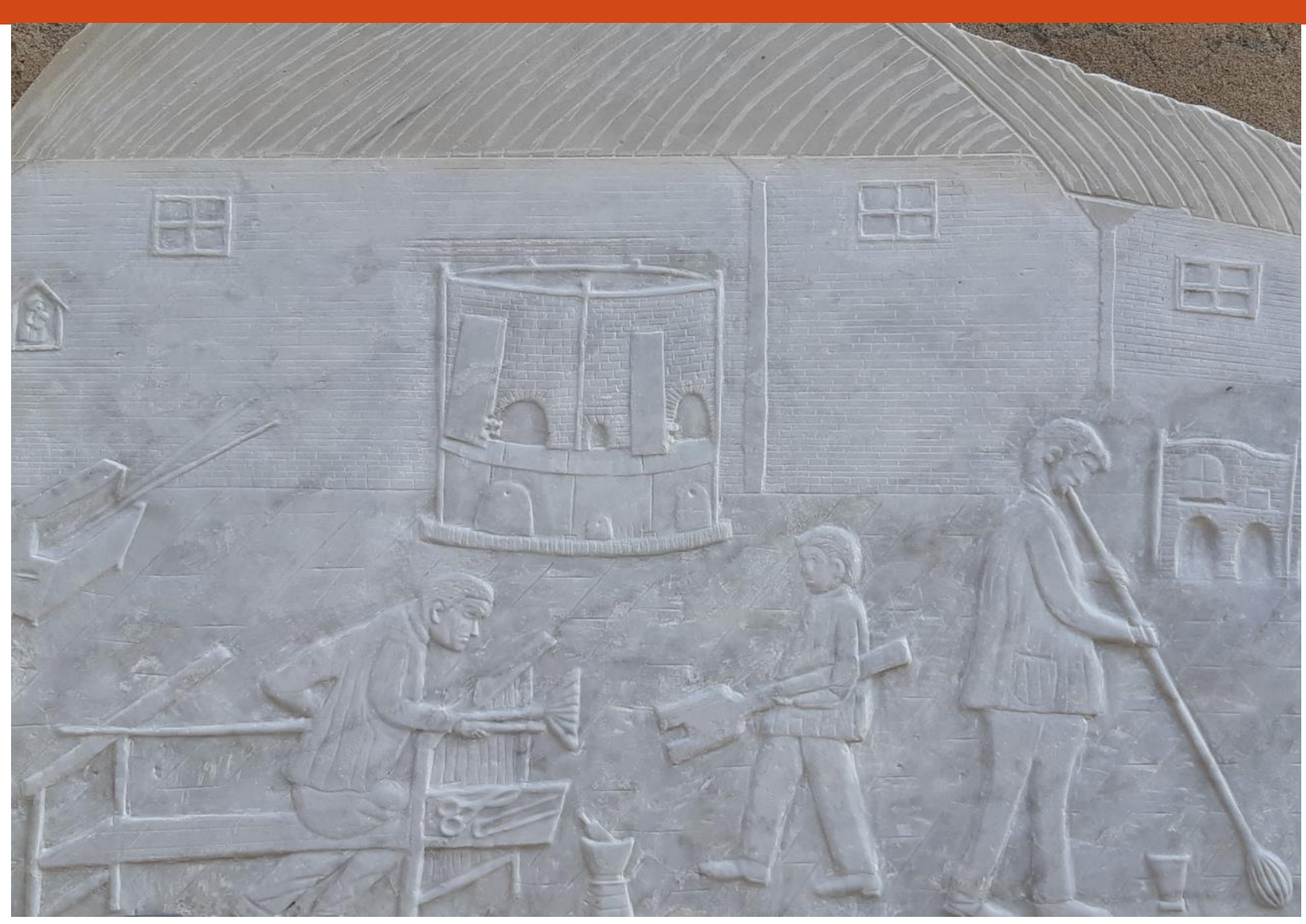

2021 ANNO VI NUMERO 12

di Paola Parravicini e Alessandro Graffi Dol https://doi.org/10.54103/2531-

$6710 / 17356$ 


\section{Autori:}

Paola Parravicini è professore associato di Economia politica nell’Università degli Studi di Milano.

Alessandro Graffi è ricercatore a tempo indeterminato in Economia politica nell'Università degli Studi di Milano

Il lavoro è frutto della ricerca comune degli autori.

Articolo soggetto a revisione tra pari a doppio cieco

Articolo ricevuto il 15.12.21 approvato il 20.12.21

\section{Introduzione}

Il dibattito accademico (e non solo!) da molti anni si sviluppa e si accende intorno alla definizione del "buon insegnante": quale dovrebbe essere il suo profilo, quale la sua formazione iniziale e quale quella in servizio ${ }^{1}$, quali competenze dovrebbe mostrare per meritare il passaggio dall'essere definito "un" insegnante a diventare "l"'insegnante che una collettività dovrebbe avere ma, soprattutto, meritarsi.

In quest'ottica si è deciso di partire dall'offerta, ossia dall'osservazione del capitale umano esistente; si è quindi ragionato sulle caratteristiche di chi ha partecipato ai percorsi per l'ottenimento dei 24 cfu in discipline antropo-psico-pedagogiche e in metodologie e tecnologie didattiche che, ad oggi, costituisce uno dei requisiti di partecipazione al concorso ordinario per l’insegnamento nella scuola secondaria di I e di II grado nonché per la partecipazione al tirocinio formativo attivo sostegno (TFA sostegno). I dati raccolti dall'esperienza nell'Università degli Studi di Milano, la dimensione, il grado di generalità dell'ateneo stesso e il posizionamento dell'Ateneo nella regione italiana che, probabilmente, è in grado di assorbire la percentuale in proporzione più elevata di aspiranti docenti, rassicurano circa la rappresentatività statistica del campione utilizzato nel rispetto di stima campionaria dei parametri, errore massimo di stima prefissato e intervalli di confidenza.

\footnotetext{
${ }^{1}$ A titolo esemplificativo e non esaustivo si veda: Allulli et al. 2014, Altet M. 1994; Altet M et al 2006; Baldacci M. 2013; Baillat G. et al 2010; Baldacci M. 2012; Bertagna G. 2019; Cappa C. et al. 2013, Corsi M 2017; Felisatti E. Tonegato P. 2013, Fiorin I. 2018; Garavaglia A., Petti L. 2017; Binanti L., Tempesta M. 2011; Grion V. 2008; Kanizsa S. 2017; Kanizsa S., Gelati M. 2009; Kanizsa S., Zaninelli F. 2020; Limone P. et al. 2016; Magnoler P. et al 2017; Margiotta U. 2017; Michelini M. 2013; Montalbetti K. 2014; Nigris E. 2004; Ostinelli G 2009; Refrigeri L. 2017; Rivoltella, P. C. 2018; Rivoltella, P. C., Rossi, P. G. 2019; Tammaro R., Petolicchio A. 2017; Vinatier L., 2008;
} 
Una prima analisi è quindi incentrata sulla possibilità di disegnare un profilo "generico" degli interessati all'insegnamento in termini di genere, età e condizione professionale; una seconda si pone invece l'obiettivo di definire l'interesse specifico del campione in termini di insegnamento per aree disciplinari. Le due analisi, complementari tra loro, consentono di giungere a delineare un profilo sufficientemente esaustivo dell'aspirante insegnante che si ritiene possa costituire un buon tessuto per alcune riflessioni "non conclusive" che, a loro volta, si auspica, possano contribuire al dibattito circa la definizione di un modello sostenibile di formazione della figura dell'insegnante.

\section{Gli aspiranti all'insegnamento e la loro carta d'identità}

L'Università degli Studi di Milano ha risposto alle indicazioni ministeriali contenute nel DM 616/2017 con l'istituzione di un percorso, annualmente bandito, rivolto sia ai laureati in ateneo e in altri atenei che agli iscritti all'ultimo anno di un corso di studio magistrale o a ciclo unico. Nell'arco temporale dei tre anni accademici di riferimento $(2017 / 18,2018 / 19,2019 / 20)$ hanno partecipato al percorso, ribattezzato in Ateneo FOR24, 7887 persone. L'analisi non riguarda gli immatricolati al percorso nel 2020-2021 (1530) e nel 2021-2022 (attualmente in corso con 1461 immatricolati)². Il motivo dell'esclusione è semplice: nell'aprile del 2020 è stato bandito il concorso per l'insegnamento e hanno potuto candidarsi solo coloro che avrebbero maturato i requisiti concorsuali entro il luglio del medesimo anno. La fotografia che questo lavoro si propone è proprio quella costituita, per il campione che la rappresenta, da coloro che si apprestano, nei prossimi mesi, a sostenere le prove concorsuali congelate dall'emergenza sanitaria.

Per quanto attiene al campione di riferimento, il rilevante numero degli immatricolati convince circa la possibilità di considerarne. In particolare, l'intero campione è costituito per l' $82,5 \%$ da laureati mentre il restante $17,5 \%$ è formato da studenti iscritti, al 2019-2020, all'ultimo anno di un corso di laurea.

Il bando di concorso ordinario per l'insegnamento nella scuola secondaria del 28 aprile 2020 ha infatti spinto verso l'alto la percentuale dei laureati nell'anno accademico in corso: entro il 31 luglio 2020, data prevista per la domanda al concorso stesso, oltre il 90\% degli iscritti al percorso lo ha concluso "per non rischiare di perdere un'opportunità".

\footnotetext{
${ }^{2}$ Sono inoltre stati esclusi dalla valutazione oltre 1000 immatricolati che, nel corso del quinquennio, hanno abbandonato il percorso senza concluderlo.
} 
Sul significato dell'insegnamento come "una" opportunità, si tornerà più avanti. Vale qui solo la pena di sottolineare come l'insegnamento sia diventato, nella considerazione sociale, un'opportunità fra diverse altre possibili, via via sempre meno probabili per ragioni soprattutto di ordine economico-sociale.

Anche l'elemento di "genere" consente alcune considerazioni; l'analisi del campione utilizzato evidenzia che il $67 \%$ degli aspiranti all'insegnamento in scuola secondaria appartiene al genere femminile. Se, da un lato, si conferma il dato "storico" che evidenzia una maggioranza femminile interessata a questa professione, dall'altro lato preoccupa, come vedremo meglio più avanti, il rinnovato interesse del genere maschile dal momento che si concentra su una fascia d'età molto alta, facendo pensare ad interesse motivato dalla necessità di ricollocamento nel mercato del lavoro.

Ed è proprio sull'età degli aspiranti che si è poi concentrata l'analisi.

Figura 1

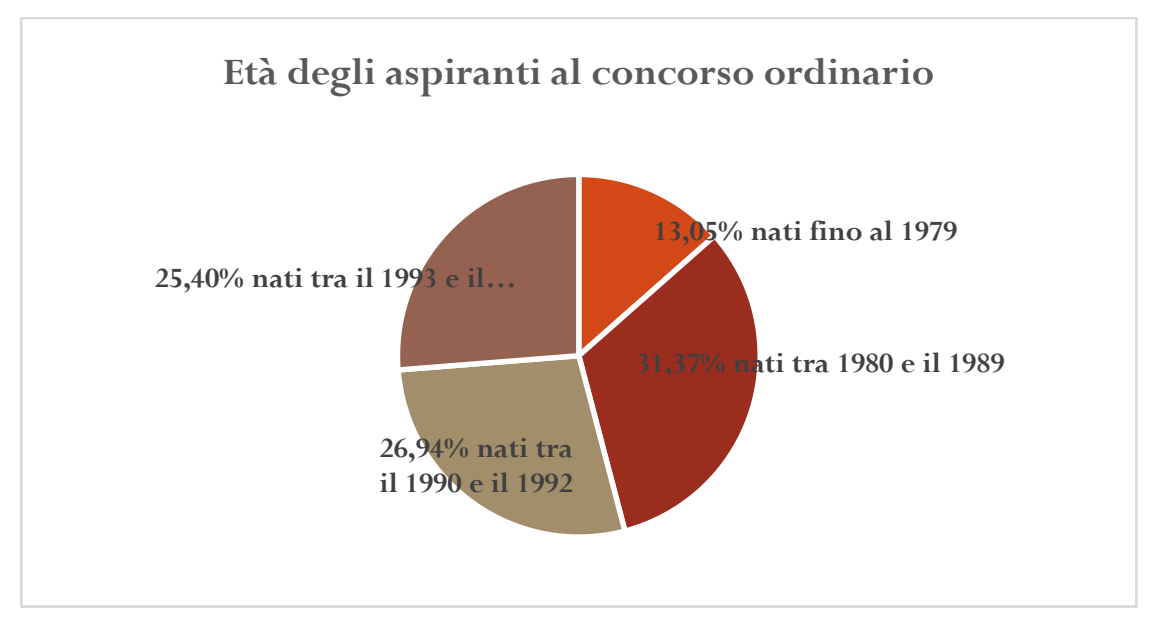

Fonte: nostre elaborazioni su dati Unimi

Come si può notare dalla Figura 1, il campione è stato suddiviso per fasce d'età.

I nati tra il 1996 e il 1997 costituiscono solo il 2,69\% ma possiamo considerare questa bassa percentuale come un dato "naturale" dal momento che corrisponde alle coorti di neolaureati o di prossimi alla laurea che quindi, ragionevolmente, non avevano ancora ottenuto tutti i requisiti utili alla partecipazione al concorso. Ciò che colpisce è invece la quota pari ad oltre il 51\% costituita dai nati fino al 1989 (nati fino al 
1979 sommati ai nati tra il 1980 e il 1989). Se la lettura del dato è corretta, infatti, si può affermare che oltre il 50\% degli aspiranti insegnanti si affaccia per la prima volta all'insegnamento ad un'età non inferiore a 33 anni. Se si considera, poi che il 15,2\% è costituito da persone con oltre 43 anni (nati fino al 1979) e che il valore modale della classe d'età di nati tra il 1980 e il 1989 è nell'anno di nascita 1983 (quindi a 39 anni), si comprende come l'età media degli insegnanti che entreranno in ruolo sarà ancora molto alta rispetto a quella dei colleghi europei. Come è noto, infatti, il problema dell'elevata età media degli insegnanti rileva da tempo in Italia (OECD 2018). In base al rapporto del 2018, in Italia gli insegnanti hanno in media 49 anni mentre nei Paesi OCSE l'età media si attesta sui 44; sempre in Italia il 48\% dei docenti di scuola ha più di 50 anni contro un dato OCSE per la medesima percentuale di 34 anni.

Figura 2

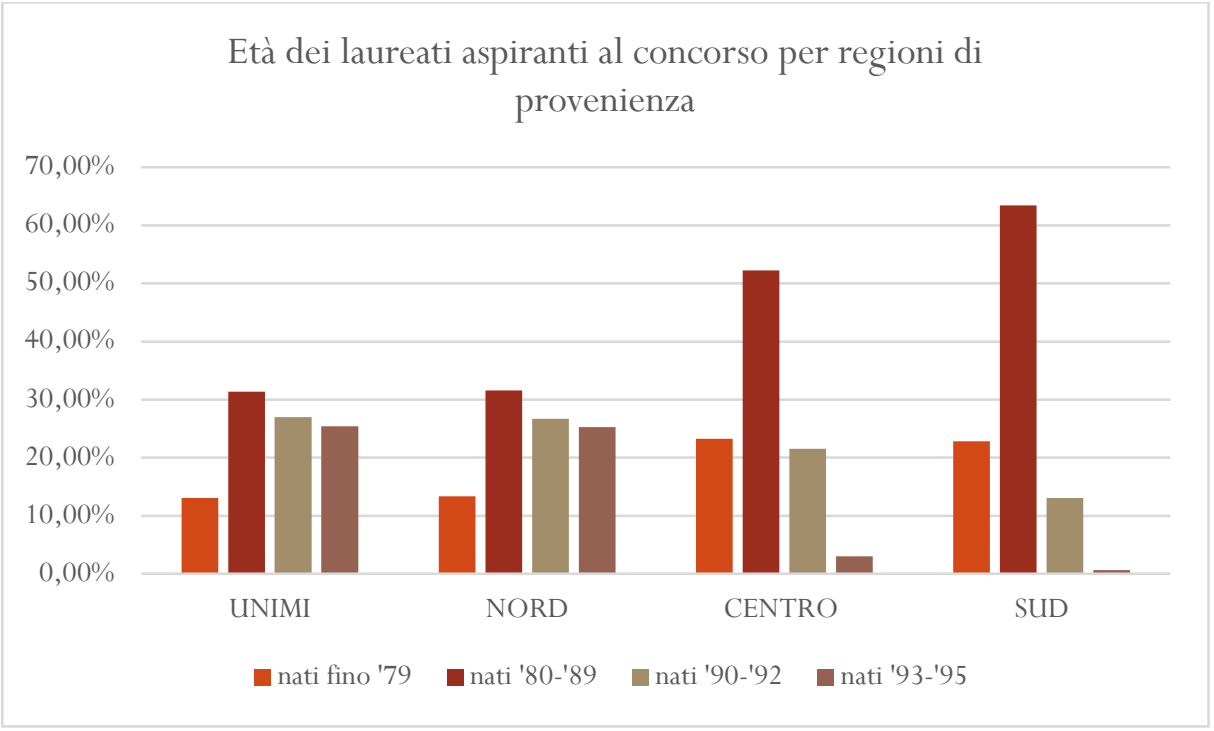

Fonte: nostre elaborazioni su dati UNIMI

Il dato che si rileva per gli iscritti al percorso FOR24 all'Università degli Studi di Milano conferma tale situazione evidenziando un ulteriore peggioramento: difficile, in queste condizioni, la rincorsa verso una riduzione dell'età media dei docenti italiani. E tale gap generazionale tra insegnanti non si può nemmeno imputare al percorso scolastico più lungo (la distanza tra il dato italiano e quello di media OCSE è ben superiore ai due anni di eventuale differenziazione massima) né a necessità di assolvimento di percorsi di 
formazione iniziale alla professione. Il percorso formativo disegnato nella legge Buona Scuola (Legge 107/2015) ha trovato tra i principali ostacoli proprio l'eccessiva articolazione del percorso formativo iniziale dell'insegnante ( 3 anni). Dal momento però che tale percorso formativo triennale non è mai venuto alla luce, si comprende come l'elevata età media dei docenti italiani abbia ragioni strutturali e non certo legate alla durata di percorsi formativi che, al momento, risultano di fatto completamente inesistenti. Al fine di comprendere meglio le cause di questo problema, sul quale si tornerà comunque anche nelle conclusioni, sono stati analizzati separatamente i laureati nell'Università degli Studi di Milano rispetto ai laureati in altre province lombarde e in altre regioni italiane che si sono rivolti all'ateneo milanese per l'ottenimento della certificazione ex DM 616/2017 (D Lgs 59/2017).

Dall'osservazione della Figura 2 risulta con chiarezza che in tutti i casi la percentuale più elevata degli aspiranti al concorso per l'insegnamento risulta costituita dai nati tra il 1980 e il $1989^{3}$. Per quanto attiene alle regioni del nord (compreso il caso di UNIMI) la percentuale dei laureati nati fino al 1989 è pari al 44\% circa, mentre per i laureati nelle regioni del centro e del sud del Paese le percentuali salgono rispettivamente al 75\% e al' $85 \%$. Questo dato, che può risultare allarmante, deve comunque essere riletto alla luce di una spiegazione non ininfluente: l'età modale per i laureati al centro e al sud del paese scende rispettivamente a 34 e 35 anni (contro i 37 del nord Italia): ciò potrebbe significare che il trasferimento dalle regioni di provenienza alla Lombardia e, ragionevolmente, a Milano è avvenuto qualche anno dopo il conseguimento della laurea probabilmente per necessità occupazionali, diverse comunque dall'insegnamento. Il dato più preoccupante è invece quello che riguarda i nati fino al 1979: potrebbe infatti rappresentare la situazione di laureati provenienti dalle regioni del Centro $(23,24 \%)$ e del Sud $(22,84 \%)$ che cercano nell'insegnamento una collocazione o, meglio, una ri-collocazione professionale. In tutti i casi, comunque, il fenomeno di aspiranti docenti che si affacciano all'insegnamento per la prima volta e con età senza dubbio elevata risulta confermato.

\footnotetext{
${ }^{3}$ Il dato sui nati nel 1996 e 1997 non è rilevante dal momento che riguarda coloro che erano (e in alcuni casi sono ancora) i soli iscritti ad un percorso di laurea in UNIMI poiché gli studenti non UNIMI sono stati ammessi al Percorso FOR24 solo se già laureati.
} 


\section{Quali discipline si desidera insegnare?}

La seconda parte dell'analisi è concentrata sulle classi di concorso alle quali gli aspiranti insegnanti potranno accedere sulla base dei corsi di studio affrontati.

Prima di esporre i principali risultati, si ritiene utile premettere alcune considerazioni.

Con la soppressione degli 8 ambiti disciplinari, avvenuta con la legge finanziaria per il 2019 (Legge n.145 del 30 dicembre 2018) e quindi modificando il decreto legislativo 59/2017 che aveva confermato i raggruppamenti delle classi di concorso in ambiti disciplinari, le procedure concorsuali finiscono di necessità con l'aumentare di numero passando da 8 a 25. Infatti, i 5 ambiti verticali della scuola secondaria di I e II grado riguardavano complessivamente 15 classi di concorso mentre i 3 ambiti orizzontali della secondaria di II grado ne raggruppavano 10.

Figura 3

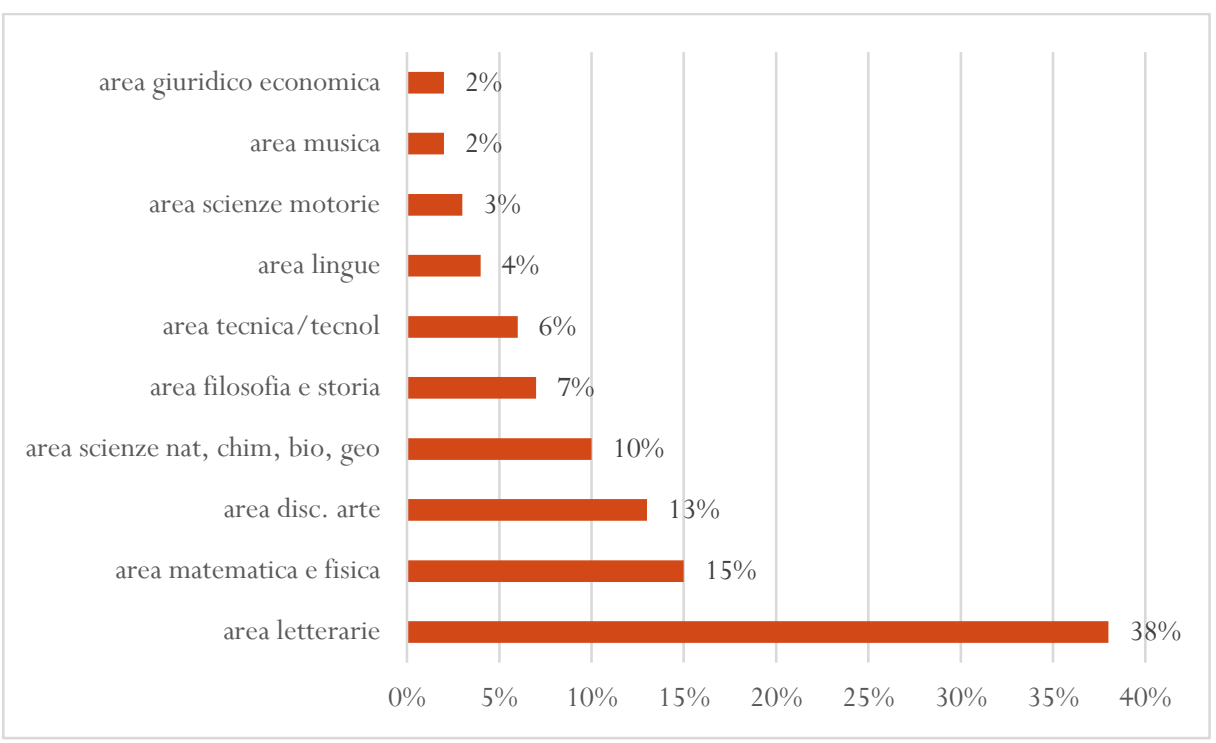

Dalla lettura dei dati a disposizione, è emerso che gli iscritti al Percorso FOR24 provengono da corsi di laurea che costituiscono titolo di accesso a 47 differenti classi di concorso. Per questo motivo è sembrato opportuno accorpare $i$ dati in dieci macroaree disciplinari che, chiaramente, non coincidono con gli 8 ambiti disciplinari che, comunque, non esistono più. 
Come si può osservare dalla Figura 3, la maggior parte degli interessati all'insegnamento sono i laureati in discipline letterarie, seguiti, ma notevole distanza, dai titolati all'insegnamento di matematica e fisica. Via via seguono le altre aree disciplinari con percentuali sempre meno rilevanti. Sono proprio le percentuali ridotte che inducono alla riflessione: come mai, in fondo, un numero così ridotto, in termini relativi s'intende, è interessato all'insegnamento?

Probabilmente, il problema nasce da lontano: questioni, mai risolte, legate al precariato storico nella scuola e all'inadeguatezza stipendiale finiscono con il rendere l'insegnamento sempre e solo un'opportunità che, se possibile, si mantiene come seconda se non terza o quarta scelta di professionalizzazione, magari da "recuperare" quando la contingenza economica diventa negativa o molto negativa.

Anche nel rapporto curato dall'Ocse (OECD, 2019), ritorna puntuale l'osservazione già nota: un insegnante di scuola primaria guadagna, in Italia, 30.403 dollari, contro una media Ocse di 31.276 dollari, per poi arrivare a fine carriera a 44.468 dollari, contro una media Ocse di 55.364 dollari.

E la distonia risulta ancora più evidente se si guardano dati relativi agli insegnanti delle secondarie di primo e secondo grado. Lo stipendio di un insegnante della scuola secondaria di I grado è pari a 32.725 dollari a fronte di una media Ocse di 34.230. E neanche l'anzianità di servizio "paga" se dopo 15 anni il confronto è tra 39.840 dollari per l'Italia e 47.675 della media Ocse. Ma, paradossalmente, per la secondaria di II grado, le distanze sono ancora maggiori: a inizio carriera un docente in Italia guadagna 32.725 dollari, contro i 35.859 della media Ocse; dopo 15 anni di servizio in ruolo, 40.952 dollari (Italia) e 49.804 dollari (media Ocse) e, a fine carriera, 51.045 (Italia) e 60.677. Ma lasciamo il commento sulla questione stipendiale degli insegnanti alle parole di Papa Francesco che, al seminario organizzato in Vaticano dalla Pontificia Accademia delle Scienze Sociali sul tema "Istruzione: il Patto globale", ha rilevato: "Davanti alle sfide dell'educazione ruolo cruciale è quello dei docenti, sempre sottopagati. La loro funzione deve essere riconosciuta e sostenuta con tutti i mezzi possibili. È necessario che abbiano a disposiz̨ione risorse nazionali, internazionali adeguate".

\section{Osservazioni non conclusive e una proposta}

Chi sono, quindi, i candidati al concorso per l'insegnamento bandito il 28 aprile 2020? Sembra proprio che si possano definire due figure principali. 
Da un lato, per il 38\% dei casi, il candidato è un laureato in discipline letterarie che, probabilmente, ha considerato l'insegnamento come la prima scelta tra le professionalità maturabili attraverso il proprio corso di laurea. Dall'altro, per il 62\% dei restanti, il candidato è un laureato per il quale l'insegnamento sembra essere, con il diminuire delle percentuali, una seconda, o terza o quarta scelta di vita professionale (Monin M., Rakoto-Roharimanana H. 2019). Come accennato nel paragrafo 2, si è persa quindi, e in modo preoccupante, la considerazione dell'insegnamento come scelta primaria di professionalizzazione e, probabilmente, la profonda crisi economica dell'ultimo quindicennio, accompagnata da una crisi profonda della politica, hanno reso "cronico" il problema. Complesso infatti è diventato rintracciare un punto di vista pubblico nella società attuale mentre a crescere velocemente, e forse anche "voracemente", è l'identità emotiva il cui sviluppo è lontano dalle categorie ideologico-filosofiche alle quali siamo stati abituati. Tutto questo, visto sotto un'altra prospettiva, si traduce in una disgregazione e frammentazione del concetto di comunità, ma la scuola è comunità.

Si è perso, in altre parole, il significato e il peso del discorso politico e questa scomparsa ha avuto conseguenze in molti ambiti; scuola, figura dell'insegnante e ruolo della sua formazione comprese.

Un discorso "politico" ha, o dovrebbe avere il senso della polis ossia della comunità considerata nella sua interezza; non è quindi rivolto a nessuno in particolare ma a ciascuno in generale. La prima necessità di chi si affacciava ad una candidatura politica (locale o nazionale che fosse) è stata quella di "definire un programma" di governo. Piano piano, ma forse è stato anche un "adagio ma non troppo", i programmi politici sono passati ad essere "in pillole" fino a sparire quasi completamente. E se anche si scrivono nessuno li legge dal momento che, se l'elettore li leggesse, si accorgerebbe facilmente della scarsa differenziazione che caratterizza gli uni dagli altri. Purtroppo però, il programma politico aveva una ratio ben precisa dal momento che costituiva la rotta sulla quale si innestavano proposte che restavano generali anche se curvate per ambiti; di qui gli interventi sull'industria, sulla sanità, sulla giustizia, sulla scuola. Tutto ciò diventava quindi coscienza socioeconomica. Non ci siamo accorti, ma è accaduto, che l'identità socioeconomica, motore e acceleratore al tempo stesso della crescita di una nazione, cedesse il posto all'identità emotiva che è, di per sé, individuale. L'identità emotiva crea, però, aggregazioni su basi profondamente differenti da quella socioeconomica: ci si aggrega, e lo si fa con connotazioni a tratti inossidabili, sulle preferenze alimentari, sulle squadre calcistiche, sull'amore per gli animali domestici, sull'affezione per il proprio 
quartiere e molte altre ancora. Nascono quindi “campanilismi emozionali” difficili da disgregare se si è persa, nel frattempo, la necessità di aggregazioni sociali più ampie, in una parola "generali". La mancanza di visione generale diventa crisi della "politica" che quindi non riesce più ad essere convincente (né avvincente): chiunque può governare, può decidere, può scegliere; non servono competenze specifiche, in pratica: non serve formazione. Ma se non servono competenze e formazione al politico perché mai dovrebbero servire ad un insegnante?

La gran parte del bilancio pubblico italiano è costituito da spese dovute (come, ad esempio, pensioni, sussidi e trasferimenti agli enti locali) e quindi i margini di manovra per scelte politiche, per esempio di investimento, si sono ridotte e l'insegnamento è diventato un ammortizzatore sociale per rispondere alla crisi economica che ha polverizzato competenze professionali, anche robuste.

Ma torniamo alla domanda iniziale: come deve essere il buon insegnante?

Le osservazioni condotte convincono circa la necessità di una rinnovata riflessione, soprattutto in una nazione che, senza dubbio più di molte altre, ha subito in questi mesi un capovolgimento epocale.

Innanzi tutto, vale la pena di sottolinearlo, la professionalità dell'insegnante va "formata" attraverso un percorso efficace ma snello che non può essere sostituito da un "pacchetto" di crediti formativi che, come è noto, possono essere costruiti in modo personalizzato dall'aspirante docente. La competenza disciplinare, che non può essere presunta, è elemento necessario e imprescindibile anche se non è sufficiente. A questa si devono collegare, e con maglie di trama e ordito ben fitte, competenze pedagogiche, ma anche psicologiche, metodologico didattiche, antropologiche e sociologiche (queste ultime dimenticate completamente dai $24 \mathrm{cfu}$ ) in un percorso che veda la convivenza sinergica di accademia e scuola. Da un lato occorre, quindi, una riflessione accademica attenta alla professionalizzazione all'insegnamento nei corsi di laurea magistrali. Ma non basta: i corsi di laurea non possono essere svuotati e nemmeno impoveriti di conoscenze, abilità e competenze per far posto ad altro. Occorre un passo in più. A questo proposito sono necessari percorsi post lauream, ben organizzati, regolamentati e, contemporaneamente, snelli. Senza dubbio molti esempi sono già stati implementati e, per alcuni aspetti, hanno mostrato criticità e storture: occorre quindi utilizzare i correttivi che l'esperienza ha trasmesso. Gli impianti, in ogni modo, anche grazie alla ricerca pedagogica, metodologica e didattico disciplinare, ci sono già. Valga per tutti la possibilità di 
considerare percorso formativo l'anno di prova che segue il superamento del concorso: questa strada, tra l'altro, non genererebbe ulteriori oneri a carico della Pubblica Amministrazione.

Nei fatti, le idee e i progetti per la formazione iniziale sono sicuramente molti ma, più spesso di quanto non si immagini, possono essere letti in una chiave convergente. Forse, un po' come per la politica, si è smesso di leggerli e, soprattutto, di richiederne fermamente l'applicazione.

D’altro canto, già nel 2005 la Commissione Europea si era occupata del tema della formazione (anche) iniziale degli insegnanti fissando principi comuni orientati a definire la professionalità dei docenti di scuola al fine di promuoverne, innalzandoli, i livelli di qualificazione (Commissione Europea 2005). Il primo principio, dopo aver evidenziato la necessità che l'insegnante europeo sia un individuo laureato, insiste sull'esigenza che la formazione iniziale sia multidisciplinare e quindi i percorsi formativi che la caratterizzano dovrebbero sia sviluppare conoscenze e competenze pedagogiche, psicologiche, antropologiche e sociologiche sia verificare e irrobustire le conoscenze disciplinari indispensabili a supportare i processi di apprendimento. Oltre a tutto ciò, si dice, i percorsi formativi non dovrebbero prescindere dalla necessaria comprensione delle dimensioni culturali e sociali dell'educazione. Forse non tutto è stato scritto ma, senza dubbio, molto lo è già; la formazione iniziale degli insegnanti non solo è indicata dalla comunità accademica ma è raccomandata dalla Commissione Europea, almeno fin dal 2005.

Ma quel che è indispensabile, infine, è una formazione iniziale stabile nella sua caratterizzazione: il continuo cambiamento nella definizione del corredo iniziale dell'insegnante è destabilizzante e fuorviante. Per essere stabile, di necessità, dovrà avere caratteristiche di flessibilità per essere in grado recepire i cambiamenti che, come abbiamo visto e vissuto, possono essere anche molto repentini.

\section{Riferimenti bibliografici}

Allulli G., Nicoli D., Magatti M., Nuovi modelli e pratiche della formazione iniziale in un quadro di sviluppo, Isfol, Franco Angeli, 2004

Altet M., La formation professionelle des enseignants, Paris, PUF, 1994

Altet M., Charlier E., Pasquay L., Perrenaud P, Formare gli insegnanti professionisti. Quali strategie, quali competenze?", Roma, Armando, 2006 
Baillat G., Niclot D., Ulma D., La formation des enseignants en Europe, Bruxelles, De Boeck, 2010

Baldacci M. (a cura di), L’insegnamento nella scuola secondaria, Napoli, Tecnodid, 2012

Baldacci M. (a cura di), La formazione dei docenti in Europa, Milano, Bruno Mondadori, 2013

Bertagna G., La formazione iniz̧iale dei docenti: una proposta epistemologica, lo scenario nazionale i suoi problemi, Nuova Secondaria, 2019

Binanti L., Tempesta M. (a cura di), La formazione del docente nella scuola europea del teræo millennio”, Lecce, Pensa, 2011

Cappa, C., Niceforo O., Palomba D., La formazione iniziale degli insegnanti in Italia, Revista española de educación comparada (22), 2013, p.139-163

Commission of the European Communities, Directorate-General for Educational and Culture, Common European Principles for Teachers Competences and Qualifications (2005 a)

Corsi M., La formazione in servizio degli insegnanti, in Domenici G. (a cura di), La formazione iniziale e in servizio degli insegnanti, Armando, Roma, 2017, pp. 225-229

Felisatti E., Tonegato P., Il laboratorio di Microteaching nel Tirocinio OnLine per la formazione iniziale degli insegnanti,Form@re, ISSN 1825-7321, Edizioni Erickson, Numero 79, Vol. 12,pp. 64-70, 2013

Fiorin I., La sfida dell'insegnamento, Mondadori, Milano, 2018

Franzini E., Iannantuoni G., Svelto F., La sfida dell'innovazione negli Atenei del dopo-crisi”, Corriere della Sera, 7 maggio 2020

Garavaglia A., Petti L., Riprogettazione del setting del laboratorio di tecnologie didattiche per la formazione iniziale degli insegnanti, in Limone P., Parmigiani D. (a cura di), Modelli pedagogici e pratiche didattiche per la formazione iniżale e in servižio degli insegnanti, Progedit - Progetti editoriali srl, Bari, 2017

Grion V., Insegnanti e formazione: realtà e prospettive, Roma, Carocci, 2008

Kanizsa S., (a cura di), Oltre il fare. I laboratori nella formazione degli insegnanti, Edizioni Junior-Bambini Srl, Reggio Emilia, 2017 
Kanizsa S., Gelati M. (a cura di), Dieci anni dell’Università dei maestri, Bergamo, Junior, 2009

Kanizsa S., Zaninelli F. (a cura di), La vita a scuola, Milano, Cortina, 2020

Limone P., Pace R., Mangione R. (a cura di), Dimensione didattica, tecnologica e organizzativa. La costruqione del processo di innovazione a scuola, Media e tecnologie per la didattica - Open Access, Franco Angeli, 2016

Magnoler P., Notti A.M., Perla L (a cura di), La professionalità degli insegnanti. La ricerca e le pratiche, Pensa multimedia, 2017

Margiotta U., La formazione iniziale degli insegnanti secondari. Per un curricolo integrato a struttura di laboratori, European Journal of Research on Education and Teaching, vol 15, n.3, 2017

\section{Michelini M., Educare il pensiero. Per la formazione dellinsegnante riflessivo, Franco Angeli, 2013}

Monin M., Rakoto-Roharimanana H. (eds), "Nuoveaux enseignants, einsegnants nouveaux: la reconversion professionelle dans le systeme educatif', Recherche et Formation, 90, 2019.

Montalbetti, K., Tra riflessione e riflessivitàIl diario di bordo nella formazione iniziale degli insegnanti: analisi di un'esperienza, “Educationaò Reflective Practices”, 2014 (1): 116-130

Nigris, E. (a cura di), La formazione degli insegnanti. Percorsi, strumenti, valutazione, Carocci editore Roma, 2004.

OECD, Education at a glance, 2020; https://www.oecd.org/education/education-at-a-glance/ 2020

OECD, Teaching and Learning International Survey - Talis, 2019

Ostinelli G., Teacher Education in Italy, Germany, England, Sweden and Finland, European Journal of Education, Volume 44, Issue 2, 2009

Refrigeri L., La economical and financial illiteracy dei docenti della scuola italiana, La professionalità degli insegnanti. La ricerca e le pratiche. Formazione, p. 405-418, Pensa Multimedia, 2017

Rivoltella, P. C., Rossi, P. G., Il corpo e la macchina. Tecnologia, cultura, educažione, Scholè, Brescia, 2019

Rivoltella, P. C., Un'idea di scuola, Scholé - Morcelliana, Brescia 2018 
Tammaro R., Petolicchio A., Formazione dei docenti e sistemi di reclutamento: un leitmotiv, Giornale Italiano

della Ricerca Educativa - Pensa MultiMedia Editore srl - anno X, n.19, dicembre 2017

Vinatier L., Altet M., Analyser et comprendre la pratique enseignant, Rennes, PUR, 2008 